Original Paper

\title{
Assessment of CIP2A And ROCK-1 EXPRession AND THEIR PROGNOSTIC VALUE IN BREAST CANCER
}

\author{
Nisreen Abdel Tawab Osman, Manal A. Khalaf, Shaymaa A.S. Ibraheem
}

Pathology Department, Faculty of Medicine, Minia University, Egypt

\begin{abstract}
Breast cancer is the most leading cause of cancer death in females worldwide. Identification of novel biomarkers for prognosis is required. Imunohistochemical evaluation of CIP2A and ROCK-1 expressions in 126 breast tissue specimens stratified as 21 ductal hyperplasias, 17 duct carcinoma in situ (DCIS) and 88 invasive carcinomas (56 invasive ductal carcinomas NST, 32 invasive lobular carcinomas) was studied. High CIP2A expression was detected in $48.9 \%$ of invasive carcinomas. CIP2A overexpression was significantly related to Nottingham prognostic index $(\mathrm{NPI})(\mathrm{p}=0.011)$, stage $(\mathrm{p}=0.01)$, ER negativity $(\mathrm{p}=0.031)$, PR negativity $(\mathrm{p}=0.048)$, and HER-2 positivity $(\mathrm{p}=0.02)$. CIP2A was significantly overexpressed in triple-negative breast cancer (TNBC) $(\mathrm{p}=0.004)$. ROCK-1 expression was detected in $54.5 \%$ of invasive carcinomas. Statistically significant associations were observed between ROCK-1 expression and NPI ( $p=0.032)$, stage $(p=0.002)$, ER negativity $(p=0.012)$, PR negativity $(p=0.023)$, HER-2 positivity $(\mathrm{p}=0.016)$, and TNBC subtype $(\mathrm{p}=0.033)$. A positive association between CIP2A and ROCK-1 expressions ( $p<0.0001)$ was documented. There was a significant association between shorter overall survival and high CIP2A and positive ROCK-1 expressions ( $\mathrm{p}<0.0001)$ and ( $\mathrm{p}<0.0001)$. CIP2A and ROCK-1 expressions could be used as markers for the poor prognosis of breast cancer.
\end{abstract}

Key words: breast cancer, CIP2A, ROCK-1, immunohistochemistry, prognosis.

\section{Introduction}

Breast cancer is the most frequently diagnosed cancer and the leading cause of cancer death in females worldwide, accounting for $11.6 \%$ of the total cancer cases and $6.6 \%$ of the total cancer deaths in 2018 [1].

In Egypt, breast cancer is the most common malignant tumor among women, constituting $32.04 \%$ of total malignancies. The frequency of breast cancer observed in different regions of Egypt was about $33.8 \%, 26.8 \%$, and $38.7 \%$ of cancer cases in upper, middle, and Lower Egypt zones, respectively [2].

Breast cancer includes several biological subtypes with a wide spectrum of clinical, pathologic and mo- lecular features resulting in different prognostic and therapeutic implications [3]. Despite the advancement in molecular studies, the prognosis and therapeutic targets of breast cancer are still depending on traditional markers such as tumor stage and hormonal receptor status $[4,5]$. Thus, identification of novel biomarkers for prognosis, prediction, and therapeutic purposes is essentially required [6].

Triple-negative breast cancer (TNBC) is a subtype of breast cancer in which the estrogen receptor together with progesterone receptor are not expressed, and human epidermal growth factor receptor 2 is not amplified or overexpressed [7]. Generally, TNBCs are more aggressive tumors with a high metastatic potential and a shorter time 
of recurrence [8]. Lacking responses for both hormonal and immunotherapeutic treatment modalities makes TNBC in a great demand for new therapeutic targets [7].

Protein phosphatase 2A (PP2A) accounts for most of the serine/threonine phosphatase activity in the cell and it acts as a tumor suppressor by inhibiting the activity of several oncogenic signaling pathways [9, 10]. Cancerous Inhibitor of PP2A (CIP2A) binds to PP2A and inhibits its phosphatase functions resulting in the tumorogenic transformation of cells [11]. CIP2A stabilizes c-Myc by inhibiting PP2A-mediated dephosphorylation of Myc at serine 62 [12]. The expression o fc-Myc rapidly induces the activation of cyclin E-cdk2 kinase activity, with the simultaneous release of $\mathrm{p} 27 \mathrm{Kip} 1$ from cdk2 complexes, which is essential for $\mathrm{G} 1 / \mathrm{S}$ transition and cell cycle progression [12].

CIP2A is overexpressed at a high frequency in a number of tumors and expression levels are independent markers for long-term outcomes in many of these tumors [11]. The clinical relevance of CIP2A overexpression as a prognostic marker has been established in various solid and hematological malignancies, including, chronic myelogenous leukemia, bladder carcinoma, non-small cell lung cancer, multiple myeloma, and endometrioid adenocarcinoma $\lfloor 13$, $14,15,16,17]$. Regarding breast cancer, CIP2A expression has been correlated with aggressiveness and also predicts a worse prognosis [18].

Alteration in the actin cytoskeleton can cause modifications in cell adhesion, contraction, migration, and invasion. Additionally, it can affect gene expression, the cell cycle, and remodeling of the extracellular matrix (ECM) [19, 20]. ROCK (Rho-associated coiled-coil kinase) belongs to the AGC family of serine/threonine protein kinases and it consists of two isoforms, ROCK-1 and ROCK-2 [21, 22]. ROCK family is mainly activated through interaction with the small Rho GTPases, including RhoA, RhoB, and RhoC [23].

The main role of the ROCK family is increasing the stabilization of actin filaments and the generation of actin-myosin contractility through phosphorylation of multiple downstream substrates. The generated contractile force influences cell behaviors including; contraction, motility, survival, and proliferation [24]. Elevated ROCK-1 and/or ROCK-2 expressions have been observed in several human cancers, in which they are associated with poor prognosis [25, 26, 27, 28]. It has also been found that there are some beneficial roles of ROCK inhibition on tumor volume, invasiveness, and metastatic potential $\{29,30]$.

Concerning breast cancer, high expression of ROCK-1 has been found to be significantly correlated with poor prognostic indicators including high grade, advanced stage, positive nodal metastasis, and shortened survival $[31,32,33]$.

To our knowledge, no previous studies have focused on the association between CIP2A and ROCK-1 in breast cancer. The aim of this work is to investigate the immunoexpression of CIP2A, and ROCK-1 in breast cancer and to correlate their expressions with different clinicopathological characteristics in order to elucidate their prognostic value and possible therapeutic implications in patients with breast cancer.

\section{Material and methods}

\section{Patients and tissue specimens}

This study included 126 formalin-fixed, paraffinembedded tissue specimens distributed as 21 ductal hyperplasia cases, 17 duct carcinoma in situ (DCIS) cases and 88 invasive carcinomas (56 invasive ductal carcinomas NST, 32 invasive lobular carcinomas). They were selected from the archives of the Department of Pathology, Minia University Hospital and Minia Oncology Center between November 2013 and November 2018 according to the availability of paraffin blocks and full clinicopathological data. This study was conducted in accordance with the 1975 Declaration of Helsinki. The histopathological categorization of tissue samples is illustrated in (Table I).

The clinicopathologic characteristics of patients of invasive breast cancers were extracted from the medical records including patients age at diagnosis, menopausal status, lymph node positivity, TNM stage, histological type, estrogen receptor (ER) status, progesterone receptor (PR) status and Her2/neu (human epidermal growth factor receptor 2). Paraffin blocks of the specimens were subjected to hematoxylin and eosin staining (HE), for reviewing the diagnosis. Overall survival was calculated in months from the date of diagnosis and ended with the time of the tumor-related death or with the last follow-up visit of the patient.

\section{Immunohistochemical procedures}

Paraffin-embedded tissue sections of $5 \mu \mathrm{m}$ thickness were deparaffinized in xylene and rehydrated through descending grades of alcohol. For antigen retrieval, the slides were heated in $10 \mathrm{mmol} / \mathrm{l}$ sodium citrate buffer ( $\mathrm{pH}$ 6.0) using a microwave oven for 20 minutes at $750 \mathrm{~W}$. Blocking of endogenous peroxidase activity was done by incubation in $0.3 \%$ hydrogen peroxide in methanol for 30 minutes.

The slides were then incubated overnight at $4^{\circ} \mathrm{C}$ in the humidity chamber with CIP2A mouse monoclonal primary antibody (clone2G10-3B5: 
Table I. The histopathological categories of study samples

\begin{tabular}{|c|c|}
\hline Tissue samples $(\mathrm{N}=126)$ & $\mathrm{N}(\%)$ \\
\hline \multicolumn{2}{|l|}{ Invasive breast cancer } \\
\hline IDC, NST & $56(44.4 \%)$ \\
\hline ILC & $32(25.4 \%)$ \\
\hline \multicolumn{2}{|l|}{ DCIS } \\
\hline Low and intermediate-grades & $7(5.6 \%)$ \\
\hline High-Grade & $10(7.9 \%)$ \\
\hline \multicolumn{2}{|l|}{ Benign lesions } \\
\hline Usual ductal hyperplasia & $14(11.1 \%)$ \\
\hline Atypical ductal hyperplasia & $7(5.6 \%)$ \\
\hline
\end{tabular}

sc-80659, 1:50 dilution, Santa Cruz Biotechnology Corporation, USA). Incubation with ROCK-1 mouse monoclonal primary antibody was done at $4^{\circ} \mathrm{C}$ overnight in the humidity chamber (clone G-6: SC-17794, 1:50 dilution, Santa Cruz Biotechnology Corporation, USA). Then, secondary biotin-conjugated antibody and the enzyme conjugate reagent was applied for 30 minutes each (Lab Vision Corporation, CA, USA).

The slides were stained with Diaminobenzidine tetrachloride (DAB) plus substrate-chromogen solution (Lab Vision Corporation, CA, USA), and then counterstained in hematoxylin. The positive control for CIP2A was human kidney tissue, while normal liver tissue was used as a positive control for ROCK-1. The replacement of the primary antibody by PBS solution was served as a negative control.

\section{Immunohistochemical assessment}

Assessing the expression of both markers was performed by three independent pathologists. Regarding CIP2A, the intensity of staining was categorized as follows; 0 (no staining), 1 (weak staining), 2 (moderate staining), or 3 (strong staining). The percentage of positive cells was categorized as follows: 1 (in $1-25 \%$ of cells), 2 (in $26-50 \%$ of cells), 3 (in $51-75 \%$ of cells), or 4 (in 76-100\% of cells). The final combined score (0-12) was obtained by multiplying both the percentage and intensity scores of each tissue section. Sections with combined scores $\leq 3$ were considered as low CIP2A expression while those having combined scores $>3$ were considered as a high expression $[15,34]$. For the ROCK-1 expression assessment, the staining intensity was scored as follows: 0 (no staining), 1 (brown) and 2 (dark brown). While for a percentage of positive cells: 0 for positive staining cells $<5 \%, 1$ for $5-25 \%, 2$ for $26-50 \%$ and 3 for above $50 \%$ cells with positive staining. The final combined score of $\geq 2$ corre- sponds to positive, while score $<2$ corresponds to negative staining [28, 35].

\section{Statistical analysis}

Data were checked, coded, entered, and analyzed by using SPSS (The Statistical Package for Social Sciences) version 17.0 software. Descriptive methods used are mean, standard deviation (SD), frequency distribution, and cross-tabulation. Significance tests: $\chi^{2}$ test was used for categorical data. Moreover, Spearman's rho correlation was performed to detect the correlation between the two markers. Statistical significance was set at $\mathrm{p}$-value $\leq 0.05$.

\section{Survival analysis}

Kaplan-Meier survival curves and log-rank test statistics were employed to evaluate overall patients' survival and their differences. Multivariate regression analysis was carried out using Cox regression to assess the specific impact of each variable on survival in the presence of other variables. Only variables of significant value from the univariate analysis were entered into the Cox regression analysis.

\section{Results}

\section{Patient characteristics}

Patients' age range was from 23 to 79 years, with a mean age (51.1 \pm 9.8$)$. Based on the ER, PR and HER2 IHC expression profile, breast cancers were stratified into 4 molecular subtypes. Luminal A: $\mathrm{ER}+$ and/or PR+, HER2-; Luminal B: ER+ and/or $\mathrm{PR}+$, HER2 + ; HER2-overexpressing: ER- and PR-, HER2 + ; Basal-like/TNBC: ER-, PR-, HER2-. Histological grading and Nottingham prognostic index (NPI) were applied for IDC cases.

\section{Immunohistochemical expression pattern of CIP2A}

The expression of CIP2A was observed in the cytoplasm of all positive cases. Normal breast tissue and all benign lesions didn't exhibit any CIP2A reactivity. Meanwhile, high CIP2A expression was detected in 3 out of 17 cases $(17.6 \%)$ of DCIS and in 43 out of 88 cases ( $48.9 \%$ ) of invasive breast cancers (Fig. 1). The difference between CIP2A expression in invasive lesions compared to DCIS areas was statistically significant ( $\mathrm{p}=0.035$; Table II).

\section{Correlation between CIP2A expression and different clinicopathologic data}

As shown in Table III, no association was found between CIP2A expression and patients' age, histologic subtype, menopausal status, laterality, or parity. 

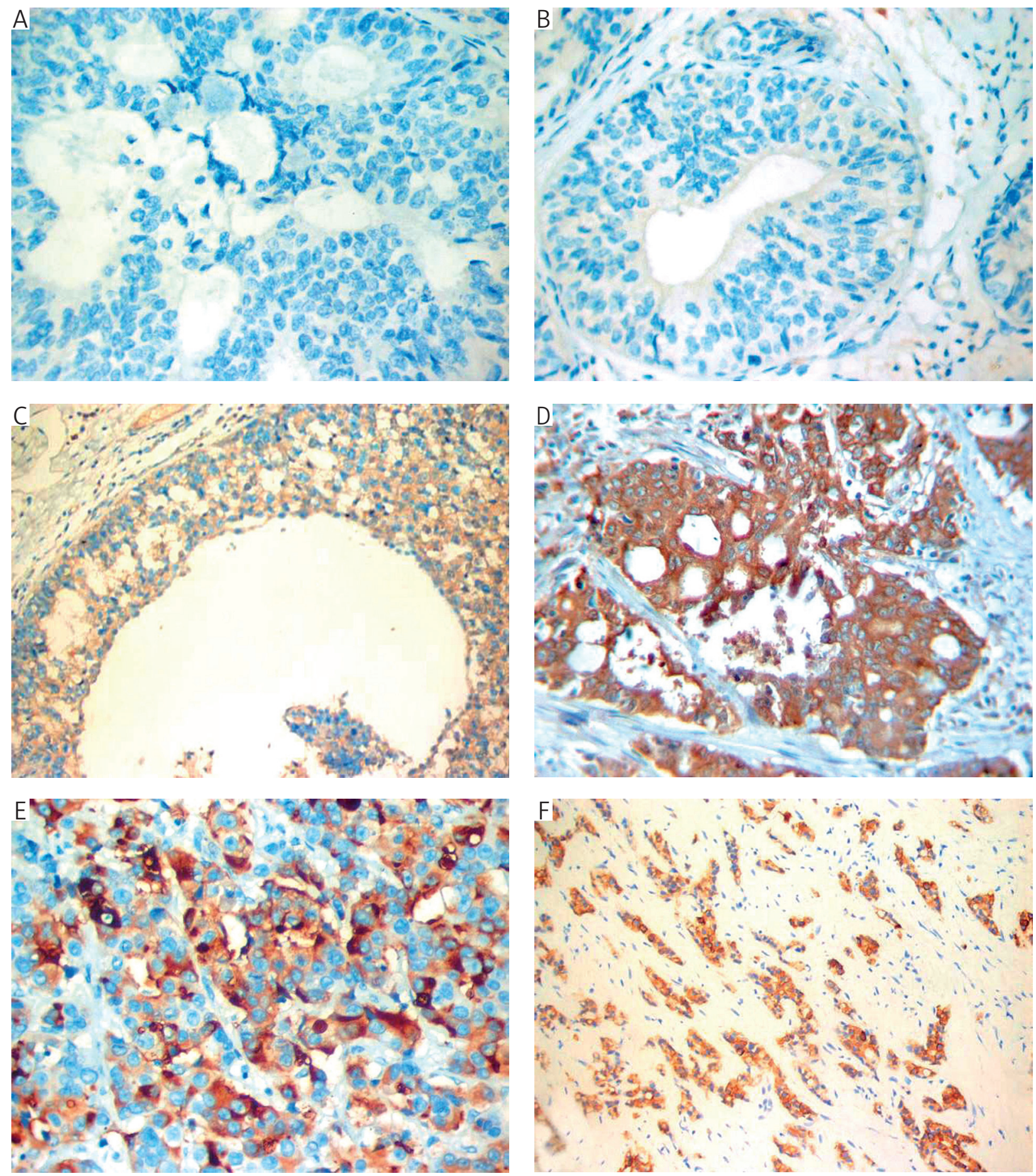

Fig. 1. Immunohistochemical expression of CIP2A in different breast lesions: A) negative CIP2A expression in atypical ductal hyperplasia; B) negative CIP2A expression in duct carcinoma in situ; C) weak positive expression of CIP2A in duct carcinoma in situ; D) strong positive expression of CIP2A in grade III invasive ductal carcinoma, NST; E) moderate positive expression of CIP2A in grade II invasive ductal carcinoma; F) moderate positive expression of CIP2A in invasive lobular carcinoma. Original magnification $400 \times$ in figures A, B, E; $200 \times$ in figures C, D, F (DAB was used as the chromogen and haematoxylin as counterstain)

CIP2A overexpression was significantly related to high histological grade $(\mathrm{p}=0.011)$, NPI $(\mathrm{p}=0.011)$, tumor size $(\mathrm{p}=0.038)$, positive lymph node metastasis $(\mathrm{p}=0.016)$, and more advanced stage $(\mathrm{p}=0.01)$. Furthermore, significant association was observed between high CIP2A expression and, ER negativity $(\mathrm{p}=0.031)$, PR negativity ( $\mathrm{p}=0.048)$, and HER-2 positivity $(\mathrm{p}=0.02)$. Also, CIP2A was significantly over expressed in TNBC compared to other molecular subtypes $(\mathrm{p}=0.004)$. 
Table II. CIP2A expression in invasive breast cancer and DCIS

\begin{tabular}{|c|c|c|c|c|}
\hline \multirow[t]{3}{*}{ Cases } & \multirow[t]{2}{*}{$\mathrm{N}=105$} & \multicolumn{2}{|c|}{ CIP2A EXPRESSION } & \multirow[t]{3}{*}{ P-VALUI } \\
\hline & & Low $(\mathrm{N}=59)$ & $\mathrm{HIGH}(\mathrm{N}=46)$ & \\
\hline & $\mathrm{N}(\%)$ & $\mathrm{N}(\%)$ & $\mathrm{N}(\%)$ & \\
\hline Invasive cancers & $88(83.8 \%)$ & $45(51.1 \%)$ & $43(48.9 \%)$ & \\
\hline DCIS & $17(16.2 \%)$ & $14(82.3 \%)$ & $3(17.6 \%)$ & $0.035 *$ \\
\hline
\end{tabular}

DCIS: duct carcinoma in situ. Test of significance: $\chi^{2}$ test. P-value $\leq 0.05$ is considered significant*

Table III. The relationship between CIP2A expression and clinicopathological characteristics of malignant invasive lesions

\begin{tabular}{|c|c|c|c|c|}
\hline \multirow{3}{*}{$\begin{array}{l}\text { CLINICOPATHOLOGICAL } \\
\text { CHARACTERISTICS }\end{array}$} & \multirow[t]{2}{*}{$\mathrm{N}=88$} & \multicolumn{2}{|c|}{ CIP2A EXPRESSION } & \multirow[t]{3}{*}{ P-VALUE } \\
\hline & & Low $(\mathrm{N}=45)$ & HIGH $(\mathrm{N}=43)$ & \\
\hline & $\mathrm{N}(\%)$ & $\mathrm{N}(\%)$ & $\mathrm{N}(\%)$ & \\
\hline \multicolumn{5}{|l|}{ Mean Age \pm SD } \\
\hline$<51.1 \pm 9.8$ & $52(59 \%)$ & $29(64.4 \%)$ & $23(53.5 \%)$ & 0.41 \\
\hline$\geq 51.1 \pm 9.8$ & $36(41 \%)$ & $16(35.6 \%)$ & $20(46.5 \%)$ & \\
\hline \multicolumn{5}{|l|}{ Histopathological Types } \\
\hline IDC, NST & $56(63.6 \%)$ & $27(60 \%)$ & $29(67.4 \%)$ & 0.61 \\
\hline ILC & $32(36.4 \%)$ & $18(40 \%)$ & $14(32.6 \%)$ & \\
\hline \multicolumn{5}{|l|}{ Menopausal Status } \\
\hline Premenopause & $50(56.8 \%)$ & $30(66.7 \%)$ & $20(46.5 \%)$ & 0.09 \\
\hline Postmenopause & $38(43.2 \%)$ & $15(33.3 \%)$ & $23(53.5 \%)$ & \\
\hline \multicolumn{5}{|l|}{ Laterality } \\
\hline Right & $29(33 \%)$ & $16(35.6 \%)$ & $13(20.2 \%)$ & \\
\hline Leftt & $46(52.3 \%)$ & $24(53.3 \%)$ & $22(51.2 \%)$ & \\
\hline Bilateral & $13(14.7 \%)$ & $5(11.1 \%)$ & $8(18.6 \%)$ & 0.6 \\
\hline \multicolumn{5}{|l|}{ Parity } \\
\hline Multipara & $74(84.1 \%)$ & $39(86.7 \%)$ & $35(81.4 \%)$ & 0.7 \\
\hline Nulipara & $14(15.9 \%)$ & $6(13.3 \%)$ & $8(18.6 \%)$ & \\
\hline Grade (for IDC, NST) & $(\mathrm{n}=56)$ & $(\mathrm{n}=27)$ & $(\mathrm{n}=29)$ & \\
\hline Grade 1 & $6(18.7 \%)$ & $5(18.5 \%)$ & $1(3.4 \%)$ & \\
\hline Grade 2 & $26(48.4 \%)$ & $16(59.3 \%)$ & $10(34.5 \%)$ & \\
\hline Grade 3 & $24(42.9 \%)$ & $6(22.2 \%)$ & $18(62.1 \%)$ & $0.011 *$ \\
\hline NPI (for IDC, NST) & $(\mathrm{n}=56)$ & $(\mathrm{n}=27)$ & $(n=29)$ & \\
\hline Good & $8(14.3 \%)$ & $6(22.2 \%)$ & $2(6.9 \%)$ & \\
\hline Moderate & $22(39.3 \%)$ & $14(51.9 \%)$ & $8(27.6 \%)$ & \\
\hline Poor & $26(48.4 \%)$ & $7(25.9 \%)$ & $19(65.5 \%)$ & $0.011 *$ \\
\hline \multicolumn{5}{|l|}{ Pathological Size } \\
\hline $\mathrm{pT} 1$ & $18(20.5 \%)$ & $13(28.9 \%)$ & $5(11.6 \%)$ & \\
\hline $\mathrm{pT} 2$ & $30(34 \%)$ & $17(37.8 \%)$ & $13(30.2 \%)$ & \\
\hline $\mathrm{pT} 3$ & $40(45.5 \%)$ & $15(33.3 \%)$ & $25(58.2 \%)$ & $0.038 *$ \\
\hline \multicolumn{5}{|l|}{ Lymph Node Status } \\
\hline N0 & $37(42 \%)$ & $25(55.6 \%)$ & $12(27.9 \%)$ & \\
\hline N1-3 & $51(58 \%)$ & $20(44.4 \%)$ & $31(72.1 \%)$ & $0.016 *$ \\
\hline
\end{tabular}


Table III. Cont.

\begin{tabular}{|c|c|c|c|c|}
\hline \multirow{3}{*}{$\begin{array}{l}\text { CLINICOPATHOLOGICAL } \\
\text { CHARACTERISTICS }\end{array}$} & \multirow[t]{2}{*}{$\mathrm{N}=88$} & \multicolumn{2}{|c|}{ CIP2A EXPRESSION } & \multirow[t]{3}{*}{ P-VALUe } \\
\hline & & Low $(\mathrm{N}=45)$ & HIGH $(\mathrm{N}=43)$ & \\
\hline & $\mathrm{N}(\%)$ & $\mathrm{N}(\%)$ & $\mathrm{N}(\%)$ & \\
\hline \multicolumn{5}{|l|}{ Stage } \\
\hline Stage I & $12(13.6 \%)$ & $9(20 \%)$ & $3(7 \%)$ & \\
\hline Stage II & $53(60.3 \%)$ & $30(66.7 \%)$ & $23(53.5 \%)$ & \\
\hline Stage III & $23(26.1 \%)$ & $6(13.3 \%)$ & $17(39.5 \%)$ & $0.01 *$ \\
\hline \multicolumn{5}{|l|}{ Estrogen receptors status } \\
\hline $\mathrm{ER}+$ & $40(45.5 \%)$ & $26(57.8 \%)$ & $14(32.6 \%)$ & \\
\hline ER- & $48(54.5 \%)$ & $19(42.2 \%)$ & $29(67.4 \%)$ & $0.031 *$ \\
\hline \multicolumn{5}{|l|}{ Progesterone receptors status } \\
\hline $\mathrm{PR}+$ & $37(42 \%)$ & $24(53.3 \%)$ & $13(30.2 \%)$ & \\
\hline $\mathrm{PR}-$ & $51(58 \%)$ & $21(46.7 \%)$ & $30(69.8 \%)$ & $0.048 *$ \\
\hline \multicolumn{5}{|l|}{ Her-2 receptors status } \\
\hline Her-2+ & $41(46.6 \%)$ & $15(33.3 \%)$ & $26(60.4 \%)$ & \\
\hline Her-2- & $47(53.4 \%)$ & $30(66.7 \%)$ & $17(39.6 \%)$ & $0.02 *$ \\
\hline \multicolumn{5}{|l|}{ Molecular subtype } \\
\hline Luminal A & $23(26.1 \%)$ & $15(33.3 \%)$ & $8(18.6 \%)$ & \\
\hline Luminal B & $21(23.9 \%)$ & $15(33.3 \%)$ & $6(14 \%)$ & \\
\hline Her $2+$ & $18(20.5 \%)$ & $9(20 \%)$ & $9(20.9 \%)$ & \\
\hline Triple-negative subtype & $26(29.5 \%)$ & $6(13.3 \%)$ & $20(46.5 \%)$ & $0.004 * *$ \\
\hline \multicolumn{5}{|l|}{ Triple-negative subtype } \\
\hline Yes & $26(29.5 \%)$ & $6(13.3 \%)$ & $20(46.5 \%)$ & \\
\hline No & $62(60.5 \%)$ & $39(86.7 \%)$ & $23(53.5 \%)$ & $0.002 * *$ \\
\hline
\end{tabular}

NPI - Nottingham prognostic index. Test of significance: $\chi^{2}$ test. P-value $\leq 0.05$ is considered significant*

Table IV. ROCK-1 expression in invasive breast cancer and DCIS

\begin{tabular}{|c|c|c|c|c|}
\hline \multirow[t]{3}{*}{ Cases } & \multirow[t]{2}{*}{$\mathrm{N}=105$} & \multicolumn{2}{|c|}{ ROCK-1 EXPRESSION } & \multirow[t]{3}{*}{ P-VALUE } \\
\hline & & Negative $(\mathrm{N}=55)$ & $\operatorname{Positive~}(\mathrm{N}=50)$ & \\
\hline & $\mathrm{N}(\%)$ & $\mathrm{N}(\%)$ & $\mathrm{N}(\%)$ & \\
\hline Invasive cancers & $88(83.8 \%)$ & $40(45.5 \%)$ & $48(54.5 \%)$ & \\
\hline DCIS & $17(16.2 \%)$ & $15(88.2 \%)$ & $2(11.8 \%)$ & $0.003 *$ \\
\hline
\end{tabular}

Immunohistochemical expression pattern of ROCK-1

ROCK-1 expression was detected in the cytoplasm. ROCK-1 expression was not detected in normal breast tissue, while 2 out of 21 cases $(9.5 \%)$ of benign lesions displayed ROCK-1 expression. Two out of 17 cases (11.8\%) of DCIS and 48 out of 88 cases $(54.5 \%)$ of invasive carcinoma were positive for ROCK-1 (Fig. 2). ROCK-1 was more obviously expressed in invasive breast cancer compared to DCIS cases ( $p=0.003$; Table IV).

\section{Correlation between ROCK-1 expression and different clinicopathologic data}

As shown in Table V, ROCK-1 expression was not associated with patient age, histologic subtypes, menopausal status, laterality, parity, or tumor size. Significant association was observed between ROCK-1 expression and high histological grade $(\mathrm{p}=0.011)$, NPI $(\mathrm{p}=0.032)$, positive lymph node metastasis $(\mathrm{p}=0.007)$, and more advanced stage $(\mathrm{p}=0.002)$. In addition, significant association was observed between ROCK-1 

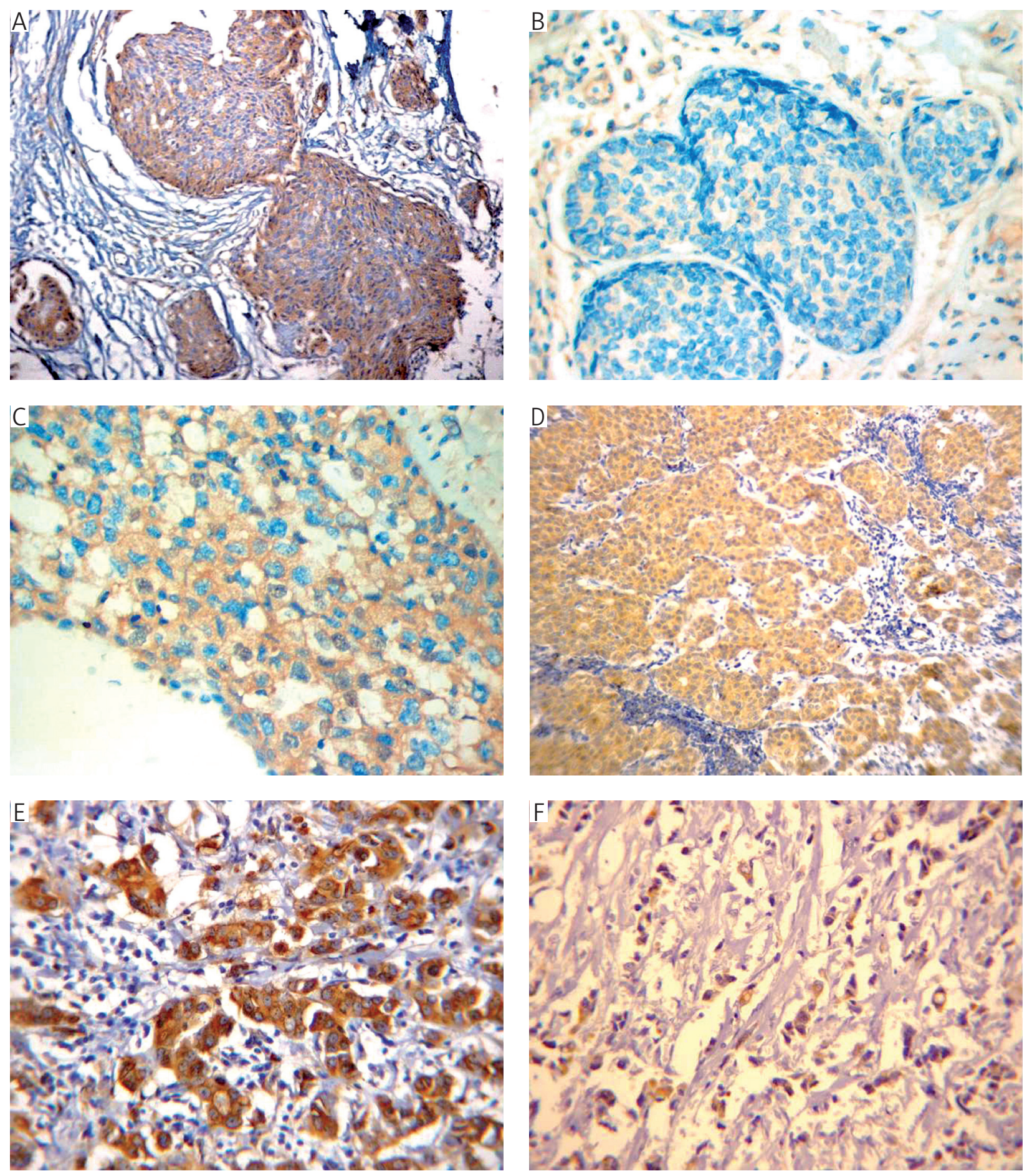

Fig. 2. Immunohistochemical expression of ROCK-1 in different breast lesions: A) positive ROCK-1 expression in atypical ductal hyperplasia; B) negative ROCK-1 expression in duct carcinoma in situ; C) positive (brown) expression of ROCK-1 in duct carcinoma in situ; D) positive (brown) expression of ROCK-1 in grade II invasive ductal carcinoma; E) positive (dark brown) expression of ROCK-1 in grade II invasive ductal carcinoma, NST; F) positive (brown) expression of ROCK-1 in invasive lobular carcinoma. Original magnification $400 \times$ in figures A, B, E; $200 \times$ in figures C, E, F (DAB was used as the chromogen and haematoxylin as counterstain) 
Table V. The relationship between ROCK-1 expression and clinicopathological characteristics of malignant invasive lesions $(\mathrm{n}=88)$

\begin{tabular}{|c|c|c|c|c|}
\hline \multirow{3}{*}{$\begin{array}{l}\text { CLINICOPATHOLOGICAL } \\
\text { CHARACTERISTICS }\end{array}$} & \multirow[t]{2}{*}{$\mathrm{N}=88$} & \multicolumn{2}{|c|}{ ROCK-1 EXPRESSION } & \multirow[t]{3}{*}{ P-VALUE } \\
\hline & & Negative $(\mathrm{N}=41)$ & $\operatorname{Positive~}(\mathrm{N}=47)$ & \\
\hline & $\mathrm{N}(\%)$ & $\mathrm{N}(\%)$ & $\mathrm{N}(\%)$ & \\
\hline \multicolumn{5}{|l|}{ Mean age \pm SD } \\
\hline$<51.1 \pm 9.8$ & $52(59 \%)$ & $28(68.3 \%)$ & $24(51.1 \%)$ & \\
\hline$\geq 51.1 \pm 9.8$ & $36(41 \%)$ & $13(31.7 \%)$ & $23(48.9 \%)$ & 0.15 \\
\hline \multicolumn{5}{|l|}{ Histopathological types } \\
\hline IDC & $56(63.6 \%)$ & $26(63.4 \%)$ & $30(63.8 \%)$ & 0.97 \\
\hline ILC & $32(36.4 \%)$ & $15(36.6 \%)$ & $17(36.2 \%)$ & \\
\hline \multicolumn{5}{|l|}{ Menopausal status } \\
\hline Premenopause & $50(56.8 \%)$ & $28(68.3 \%)$ & $22(46.8 \%)$ & 0.07 \\
\hline Postmenopause & $38(43.2 \%)$ & $13(31.7 \%)$ & $25(53.2 \%)$ & \\
\hline \multicolumn{5}{|l|}{ Laterality } \\
\hline Right & $29(33 \%)$ & $11(36.7 \%)$ & $18(38.3 \%)$ & \\
\hline Leftt & $46(52.3 \%)$ & $25(61 \%)$ & $21(44.7 \%)$ & 0.31 \\
\hline Bilateral & $13(14.7 \%)$ & $5(12.3 \%)$ & $8(17 \%)$ & \\
\hline \multicolumn{5}{|l|}{ Parity } \\
\hline Multipara & $74(84.1 \%)$ & $34(82.9 \%)$ & $39(85.1 \%)$ & 0.78 \\
\hline Nulipara & $14(15.9 \%)$ & $7(17.1 \%)$ & $7(14.9 \%)$ & \\
\hline Grade (only for IDC, NST) & $(n=56)$ & $(\mathrm{n}=26)$ & $(\mathrm{n}=30)$ & \\
\hline Grade 1 & $6(18.7 \%)$ & $5(19.2 \%)$ & $1(3.3 \%)$ & \\
\hline Grade 2 & $26(48.4 \%)$ & $15(57.7 \%)$ & $11(36.7 \%)$ & $0.011 *$ \\
\hline Grade 3 & $24(42.9 \%)$ & $6(23.1 \%)$ & $18(60 \%)$ & \\
\hline NPI (only for IDC, NST) & $(n=56)$ & $(\mathrm{n}=26)$ & $(\mathrm{n}=30)$ & \\
\hline Good & $8(14.3 \%)$ & $7(26.9 \%)$ & $1(3.3 \%)$ & \\
\hline Moderate & $22(39.3 \%)$ & $10(38.5 \%)$ & $12(40 \%)$ & $0.032 *$ \\
\hline Poor & $26(48.4 \%)$ & $9(34.6 \%)$ & $17(56.7 \%)$ & \\
\hline \multicolumn{5}{|l|}{ Pathological size } \\
\hline $\mathrm{pT} 1$ & $18(20.5 \%)$ & $10(24.3 \%)$ & $8(17 \%)$ & \\
\hline $\mathrm{pT} 2$ & $30(34 \%)$ & $14(34.1 \%)$ & $16(33.1 \%)$ & 0.65 \\
\hline pT3 & $40(45.5 \%)$ & $17(41.6 \%)$ & $23(48.9 \%)$ & \\
\hline \multicolumn{5}{|l|}{ Lymph node status } \\
\hline N0 & $37(42 \%)$ & $24(58.4 \%)$ & $13(27.7 \%)$ & $0.007 * *$ \\
\hline $\mathrm{N} 1-3$ & $51(58 \%)$ & $17(41.6 \%)$ & $34(72.3 \%)$ & \\
\hline \multicolumn{5}{|l|}{ Stage } \\
\hline Stage I & $12(13.6 \%)$ & $10(24.3 \%)$ & $2(4.3 \%)$ & \\
\hline Stage II & $53(60.3 \%)$ & $26(63.4 \%)$ & $27(57.4 \%)$ & $0.002 *$ \\
\hline Stage III & $23(26.1 \%)$ & $5(12.3 \%)$ & $18(38.3 \%)$ & \\
\hline \multicolumn{5}{|l|}{ Estrogen receptors status } \\
\hline $\mathrm{ER}+$ & $40(45.5 \%)$ & $25(61 \%)$ & $15(31.9 \%)$ & $0.012 *$ \\
\hline $\mathrm{ER}-$ & $48(54.5 \%)$ & $16(39 \%)$ & $32(68.1 \%)$ & \\
\hline
\end{tabular}


Table V. Cont.

\begin{tabular}{|c|c|c|c|c|}
\hline \multirow{3}{*}{$\begin{array}{l}\text { CLINICOPATHOLOGICAL } \\
\text { CHARACTERISTICS }\end{array}$} & \multirow{2}{*}{$\mathrm{N}=88$} & \multicolumn{2}{|c|}{ ROCK-1 EXPRESSION } & \multirow[t]{3}{*}{ P-VALUE } \\
\hline & & Negative $(\mathrm{N}=41)$ & Positive $(\mathrm{N}=47)$ & \\
\hline & $\mathrm{N}(\%)$ & $\mathrm{N}(\%)$ & $\mathrm{N}(\%)$ & \\
\hline \multicolumn{5}{|l|}{ Progesterone receptors status } \\
\hline $\mathrm{PR}+$ & $37(42 \%)$ & $23(56.1 \%)$ & $14(29.8 \%)$ & $0.023 *$ \\
\hline $\mathrm{PR}-$ & $51(58 \%)$ & $18(43.9 \%)$ & $33(70.1 \%)$ & \\
\hline \multicolumn{5}{|l|}{ Her-2 receptors status } \\
\hline Her-2+ & $41(46.6 \%)$ & $13(31.7 \%)$ & $28(59.6 \%)$ & $0.016 *$ \\
\hline Her-2- & $47(53.4 \%)$ & $28(68.3 \%)$ & $19(40.4 \%)$ & \\
\hline \multicolumn{5}{|l|}{ Molecular subtype } \\
\hline Luminal A & $23(26.1 \%)$ & $13(31.7 \%)$ & $10(21.3 \%)$ & \\
\hline Luminal B & $21(23.9 \%)$ & $13(31.7 \%)$ & $8(17 \%)$ & $0.033 *$ \\
\hline Her-2+ & $18(20.5 \%)$ & $9(22 \%)$ & $9(19.1 \%)$ & \\
\hline Triple-negative subtype & $26(29.5 \%)$ & $6(14.6 \%)$ & $20(42.6 \%)$ & \\
\hline \multicolumn{5}{|l|}{ Triple-negative subtype } \\
\hline Yes & $26(29.5 \%)$ & $7(17.1 \%)$ & $19(40.4 \%)$ & $0.031 *$ \\
\hline No & $62(60.5 \%)$ & $34(82.9 \%)$ & $28(59.6 \%)$ & \\
\hline
\end{tabular}

NPI - Nottingham prognostic index. Test of significance: $\chi^{2}$ test. P-value $\leq 0.05$ is considered significant*

Table VI. Multivariate analyses for overall survival in 88 patients with malignant invasive lesions

\begin{tabular}{lcccc}
\hline VARIABLES & B & SE & P-VALUE & HR $(95 \%$ CI $)$ \\
\hline Histopathological type & 0.771 & 0.262 & 0.003 & $2.162(1.292-3.616)$ \\
\hline Grade & 0.507 & 0.319 & 2.535 & $1.661(0.889-1.752)$ \\
\hline NPI (only for IDC) & 0.169 & 0.200 & 0.399 & $1.184(0.800-1.752)$ \\
\hline Pathological size & 0.078 & 0.196 & 0.689 & $1.081(0.737-1.586)$ \\
\hline Stage & 0.275 & 0.195 & 0.159 & $1.317(0.898-1.930)$ \\
\hline Estrogen receptor status & -0.383 & 0.394 & 0.330 & $0.682(0.315-1.475)$ \\
\hline Progesterone receptor status & -0.044 & 0.367 & 0.904 & $0.957(0.466-1.963)$ \\
\hline Triple-negative subtype & 0.157 & 0.320 & 0.623 & $0.706(0.152-0.604)$ \\
\hline CIP2A expression & -0.349 & 0.345 & 0.312 & $0.706(0.359-1.388)$ \\
\hline ROCK-1 expression & -1.194 & 0.352 & 0.001 & $0.303(0.152-0.604)$ \\
\hline B-rat
\end{tabular}

$B$ - regression coefficient; SE - standard error; $H R$ - bazard ratio; $C I$ - confidence interval; NPI-Nottingham prognostic index; p-value $\leq \_.05$ is considered significant. Cox regression test is used

expression and, ER negativity ( $\mathrm{p}=0.012)$, PR negativity ( $\mathrm{p}=0.023)$, HER-2 positivity $(\mathrm{p}=0.016)$, and TNBC subtype $(p=0.033)$.

\section{Association between CIP2A and ROCK-1 expressions}

We further evaluated the association between CIP2A and ROCK-1 expressions in invasive breast carcinoma cases. Our results showed positive association between CIP2A and ROCK-1 expressions $\left(r_{s}=0.594 ; p<0.0001\right)($ Fig. 3).

\section{Correlation between CIP2A and ROCK-1 expressions and survival of patients}

Thirty two patients (18.25\% of all cases studied; $36.36 \%$ of invasive cancer cases) died during 5-year follow-up. The median overall survival period was 41.5 months (range 14-60 months). Using Kaplan- Meier method and log-rank test, there was a significant association between shorter overall survival of breast cancer and larger tumor size $(\mathrm{p}=0.005)$, tumor subtype $(\mathrm{p}=0.005)$, histologic grade $(p=0.030)$, high stage $(p=0.027)$, 


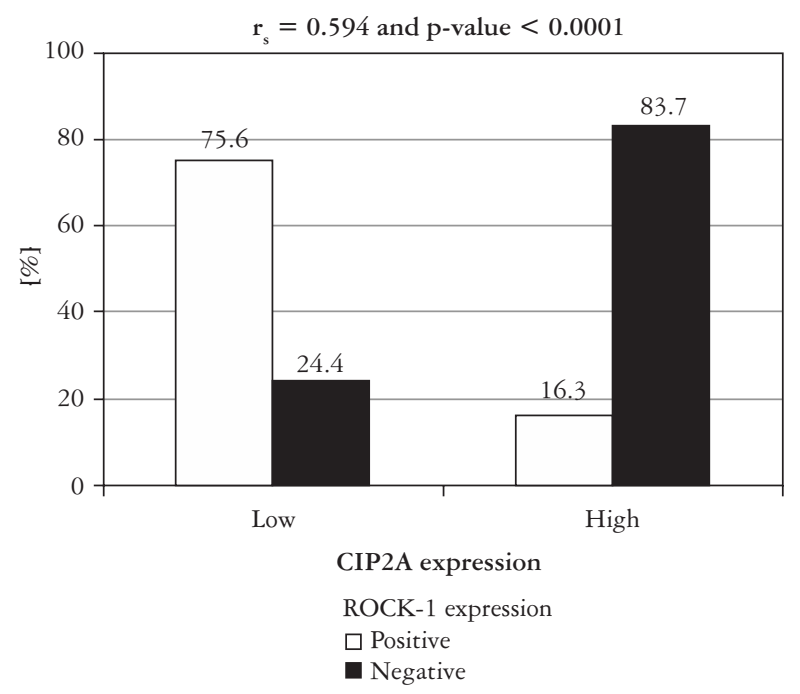

Test of significance: Spearman's rho correlation. P-value $<0.05$ is considered significant

Figure 3. Relation between CIP2A and ROCK-1 expression in invasive breast carcinoma cases $(\mathrm{n}=88)$

NPI ( $\mathrm{p}=0.003)$, estrogen receptor negativity $(\mathrm{p}<0.0001)$, progesterone receptor negativity $(\mathrm{p}=0.029)$, triple-negative subtype $(\mathrm{p}=0.048)$, high CIP2A expression $(\mathrm{p}<0.0001)$ and high ROCK-1 expression $(\mathrm{p}<0.0001)$. Postoperative mortality was observed in $18 / 56$ patients with invasive ductal carcinoma, NST (32.14\%) and in 14/32 patients with invasive lobular carcinoma (43.75\%) during 5-year follow-up. On the other hand, no significant association was found between overall survival of breast cancer cases and both age $(p=0.733)$ and HER-2 expression ( $\mathrm{p}=0.723$ ).

In multivariate analysis, only ductal tumor subtype and ROCK-1 expression were independent predictor factors for reduced overall survival $(p=0.0003$; 0.001 respectively; as shown in Table VI).

\section{Discussion}

The present study has analyzed the expression patterns of CIP2A and ROCK-1 in different breast lesions. High cytoplasmic expression of CIP2A was detected in 43/88 (48.9\%) invasive carcinoma cases. In DCIS, 3/17 (17.6\%) cases displayed high CIP2A expression. We have reported significant CIP2A overexpression in both invasive breast carcinoma and DCIS cases when compared to benign lesions. Furthermore, adjacent normal breast tissues did not display any CIP2A expression. This was in line with another study that reported high cytoplasmic expression of CIP2A protein in 39\% of 33 breast carcinomas without expression in normal mammary tissue [36]. Therefore, the previous findings can suggest a possible association of
CIP2A overexpression with cancer progression and aggressiveness.

In the present study, CIP2A overexpression was significantly related to high histological grade, NPI, positive lymph node metastasis, advanced stage, and shorter overall survival. Furthermore, a significant association was observed between high CIP2A expression and HER-2 positivity, ER and PR negativity. Many studies reported association of high CIP2A expression with poor prognostic factors [36, 37, 38, 39] and recurrence after treatment [18].

Concerning molecular subtypes, CIP2A was significantly overexpressed in TNBC. This finding was in agreement with a previous study that demonstrated overexpression of CIP2A mRNA and protein in the TNBC cell line and tissue compared with receptor-positive cell lines and cells [39]. Moreover, CIP2A depletion in TNBC cell lines resulted in inhibition of proliferation and invasion, on one hand, and induction of apoptosis and autophagy on the other hand. Another study showed that increased CIP2A expression was significantly related to basal-like and HER2 + breast cancers [40].

Our results displayed more positive expression of ROCK-1 in invasive breast cancer than both DCIS and benign lesions to statistically significant levels $(p=0.003)$. In accordance with our results, previous studies showed no ROCK-1 expression in normal breast tissues, while high ROCK-1 expression was detected especially in patients with metastasis [33, $41,42]$. They also concluded that ROCK-1 inhibition can decrease cell migration, proliferation, and metastasis $[43,44]$. Taken together, the previous findings can confirm the obvious role of ROCK-1 in promoting breast cancer metastasis.

We found significant associations between ROCK-1 expression and higher histological grade, NPI, positive lymph node metastasis, advanced stage, and reduced overall survival. Furthermore, we demonstrated significant associations between ROCK-1 expression and ER negativity, PR negativity, HER-2 positivity and also with TNBC. Many publications have demonstrated significant association of ROCK-1 expression with disadvantageous prognostic parameters in breast cancer $[33,41,42,45]$.

In our study, the association between CIP2A and ROCK-1 expressions in breast cancer was studied and the positive association between these proteins could suggest possible link between both CIP2A and ROCK-1 in progression of breast cancer. This finding needs future research work to confirm it.

The possible explanation for such positive association between CIP2A and ROCK-1 overexpression in breast cancer could be postulated based on their roles in cell cycle progression. On one hand, ROCK signaling is implicated in cell cycle progression through regulation of several cell cycle regulatory proteins by 
different mechanisms. One of these mechanisms is through reduction of p27 levels, which in turn stimulates G1/S cell cycle progression [46]. Moreover, Rho activity was found to phosphorylate c-Myc and promote its stability and transcriptional activity in breast and prostate cancers [43, 47]. On the other hand, it has previously been shown that CIP2A inhibits c-Myc dephosphorylation and stabilizes its activity, resulting in release of p27 from cyclin E-cdk2 kinase complexes, which is needed for G1/S transition and cell cycle progression [12]. Also, CIP2A knockdown resulted in increased p27 level and thus resulted in arrest of cell cycle in TNBC [39].

Regarding the role of targeting CIP2A towards the treatment of breast cancer, the results of previous studies confirmed that Arctigenin inhibits triple-negative breast cancers proliferation, progression and invasion by targeting CIP2A to reactivate protein phosphatase 2A [48]. Moreover, CIP2A mediate bortezomib-induced apoptosis in TNBC cells. Thus, CIP2A may be a potential therapeutic target in TNBC [49].

Previous studies reported that ROCK1 expression was decreased by treatment with EGFR inhibitor, MEK inhibitor and Integrin $\beta 1$ function blocking antibody. Two ROCK inhibitors (Y-27632 and Fasudil, $30 \mu \mathrm{M}$ ) caused a decrease in both EGFR and Integrin $\beta 1$ protein levels. Furthermore, the ROCK inhibitor Y-26735 reduced the levels of GLUT3 and LDHA proteins in breast cancer cells. These observations suggest that the ROCK signaling pathways are integrated with other signaling pathways. Disruption of these pathways leads to a malignant phenotype observed in breast cancer cells $[50,51]$. Thus ROCK inhibitors may be valuable for prevention of invasion and metastasis [52].

\section{Conclusions}

In summary, our research demonstrates that high CIP2A and ROCK-1 expressions are associated with breast cancer progression. Only, ROCK-1 expression was associated with tumor aggressiveness, poor prognosis and decreased overall survival. Our results indicate that CIP2A together with ROCK-1 might be promising predictive biomarkers and can be possible targets for future therapeutic regimens in breast cancer treatment. Further studies are required to understand in depth the underlying molecular and cellular mechanisms of CIP2A and ROCK-1 in breast cancer.

The authors declare no conflict of interest.

\section{References}

1. Bray F, Ferlay J, Soerjomataram I, et al. Global Cancer Statistics 2018: GLOBOCAN Estimates of Incidence and Mortality Worldwide for 36 Cancers in 185 Countries. Cancer J Clin 2018; 68: 394-424.
2. Ibrahim AS, Khaled HM, Mikhail NH, et al. Cancer Incidence in Egypt: Results of the National Population-Based Cancer Registry Program. J Cancer Epidemiol 2014; 2014: ID 437971.

3. Greenlee RT, Mukesh BN, Onitilo AA, Engel J. Breast Cancer Subtypes Based on ER/PR and Her2 Expression: Comparison of Clinicopathologic Features and Survival. Clin Med Res 2009; 7: 4-13.

4. Singletary SE, Allred C, Ashley P, et al. Staging system for breast cancer: revisions for the 6th edition of the AJCC Cancer Staging Manual. Surg Clin North Am 2003; 83: 803-819.

5. Bast RC, Ravdin P, Hayes DF, et al. 2000 Update of recommendations for the use of tumor markers in breast and colorectal cancer: clinical practice guidelines of the American society of clinical oncology. J Clin Oncol 2001; 19: 1865-1878.

6. Morin PJ. Claudin proteins in human cancer: promising new targets for diagnosis and therapy. Cancer Res 2005; 65: 9603 9606.

7. Marotti JD, de Abreu FB, Wells WA, Tsongalis GJ. TripleNegative Breast Cancer, Next-Generation Sequencing for Target Identification. Am J Pathol 2017; 187: 2133-2138.

8. Kaplan HG, Malmgren JA, Atwood M. T1N0 triple negative breast cancer: risk of recurrence and adjuvant chemotherapy. Breast J 2009; 15: 454-460.

9. Westermarck J, Hahn WC. Multiple pathways regulated by the tumor suppressor PP2A in transformation. Trends Mol Med 2008; 14: 152-160.

10. Khanna A, Pimanda JE, Westermarck J. Cancerous Inhibitor of Protein Phosphatase 2A, an Emerging Human Oncoprotein and a Potential Cancer Therapy Target. Cancer Res 2013; 73 : 6548-6553.

11. Junttila MR, Puustinen P, Niemelä M, et al. CIP2A inhibits PP2A in human malignancies. Cell 2007; 130: 51-62.

12. Arnold HK, Sears RC. Protein phosphatase 2 A regulatory subunit B56alpha associates with c-myc and negatively regulates c-myc accumulation. Mol Cell Biol 2006; 26: 2832-2844.

13. Lucas CM, Harris RJ, Giannoudis A, et al. Cancerous inhibitor of PP2A (CIP2A) at diagnosis of chronic myeloidleukemia is a critical determinant of disease progression. Blood 2011; 117 : 6660-6668.

14. Pang X, Fu X, Chen S, et al. Overexpression of CIP2A promotes bladder cancer progression by regulating EMT. Clin Transl Oncol 2016; 18: 289-295.

15. Cha G, Xu J, Xu X, et al. High expression of CIP2A protein is associated with tumor aggressiveness in stage I-III NSCLC and correlates with poor prognosis. Onco Targets Ther 2017; 10: 5907-5914.

16. Liu X, Cao W, Qin S, et al. Overexpression of CIP2A is associated with poorprognosis in multiplemyeloma. Signal Transduct Target Ther 2017; 2: 17013 .

17. Yu N, Zhang T, Zhao D, et al. CIP2A is overexpressed in human endometrioid adenocarcinoma and regulates cell proliferation, invasion and apoptosis. Pathol Res Pract 2018; 214: 233-239.

18. Baldacchino S, Wastall LM, Saliba C, et al. CIP2A expression predicts recurrences of tamoxifen-treated breast cancer. Tumour Biol 2017; 39: 1010428317722064.

19. Singh P, Carraher C, Schwarzbauer JE. Assembly of fibronectin extracellular matrix. Annu Rev Cell Dev Biol 2010; 10: 397-419.

20. Skarp KP, Vartiainen MK. Actin on DNA-An ancient and dynamic relationship. Cytoskeleton 2010; 67: 487-495.

21. Matsui T, Amano M, Yamamoto T, et al. Rho-associated kinase, a novel serine/threonine kinase, as a putative target for small GTP binding protein Rho. EMBO J 1996; 15: 2208-2216.

22. Julian L, Olson MF. Rho-associated coiled-coil containing kinases (ROCK): structure, regulation, and functions. Small GTPases 2014; 5: e29846. 
23. Blumenstein L, Ahmadian MR. Models of the cooperative mechanism for Rho effector recognition: implications for RhoA-mediated effector activation. J Biol Chem 2004; 279: 53419-53426

24. Benitah SA, Valeron PF, van Aelst L, et al. Rho GTPases in human cancer: an unresolved link to upstream and downstream transcriptional regulation. Biochim Biophys Acta 2004; 1705: 121-132.

25. Kamai T, Tsujii T, Arai K, et al. Significant association of Rho/ ROCK pathway with invasion and metastasis of bladder cancer. Clin Cancer Res 2003; 9: 2632-2641.

26. Abe H, Kamai T, Tsujii T, et al. Possible role of the RhoC/ ROCK pathway in progression of clear cell renal cell carcinoma. Biomed Res 2008; 29: 155-161.

27. Akagi EM, Lavorato-Rocha AM, Maia B de M, et al. ROCK1 as a novel prognostic marker in vulvar cancer. BMC Cancer 2014; 14: 822 .

28. Wu YJ, Tang Y, Li ZF, et al. Expression and significance of Rac1, Pak1 and Rock1 in gastric carcinoma. Asia Pac J Clin Oncol 2014; 10: e33-e39.

29. Xue F, Takahara T, Yata Y, et al. Blockade of Rho/Rho-associated coiled coil-forming kinase signaling can prevent progression of hepatocellular carcinoma in matrix metalloproteinasedependent manner. Hepatol Res 2008; 38: 810-817.

30. Jeong KJ, Park SY, Cho KH, et al. The Rho/ROCK pathway for lysophosphatidic acid-induced proteolytic enzyme expression and ovarian cancer cell invasion. Oncogene 2012; 31: 4279-4289.

31. Liu S, Goldstein RH, Scepansky EM, Rosenblatt M. Inhibition of rho- associated kinase signaling prevents breastcancer metastasis to human bone. Cancer Res 2009; 69: 8742-8751.

32. Patel RA, Forinash KD, Pireddu R, et al. RKI- 1447 is a potent inhibitor of the Rho associatedROCK kinases with anti-invasive and antitumoractivities in breast cancer. Cancer Res 2012; 72: 5025-5034.

33. Bottinoa J, Gelaleti GB, Maschioa LB, et al. Immuno expression of ROCK-1 and MMP-9 as prognostic markers in breast cancer. Acta Histochemica 2014; 116: 1367-1373.

34. Dong QZ, Wang Y, Dong XJ, et al. CIP2A is overexpressed in non-small cell lung cancer and correlates with poor prognosis. Ann Surg Oncol 2011; 18: 857-865.

35. Wang JX, Zhou YN, Zou SJ, et al. Correlations of P21 activated kinase 1 expression to clinicopathological features of gastric carcinoma and patients prognosis. Chin J Cancer 2010; 29: 649-654.

36. Côme C, Laine A, Chanrion M, et al. CIP2A is associated with human breast cancer aggressivity. Clin Cancer Res 2009; 15: 5092-5100.

37. Niemelä M, Kauko O, Sihto H, et al. CIP2A signature reveals the MYC dependency of CIP2A-regulated phenotypes and its clinical association with breast cancer subtypes. Oncogene 2012; 31: 4266-4278.

38. Yu G, Liu G, Dong J, Jin Y. Clinical implications of CIP2A protein expression in breast cancer. Med Oncol 2013; 30: 524.

39. Liu H, Qiu H, Song Y, et al. Cip2a promotes cell cycle progression in triple-negative breast cancer cells by regulating the expression and nuclear export of p27Kip1. Oncogene 2017; 36: 1952-1964.

40. Li S, Feng TT, Guo Y, et al. Expression of cancerous inhibitor of protein phosphatase $2 \mathrm{~A}$ in human triple negative breast cancer correlates with tumor survival, invasion and autophagy. Oncol Lett 2016; 12: 5370-5376.

41. Gilkes DM, Xiang L, Lee SJ, et al. Hypoxia-inducible factors mediate coordinated RhoAROCK1expression and signaling in breast cancer cells. Proc Natl Acad Sci U S A 2014; 111: E384-E393.
42. Lane J, Martin TA, Watkins G, et al. The expression and prognostic value of ROCK I and ROCK II and their role in human breast cancer. Int J Oncol 2008; 33: 585-593.

43. Liu S, Goldstein RH, Scepansky EM, Rosenblatt M. Inhibition of Rho-Associated Kinase Signaling Prevents Breast Cancer Metastasis to Human Bone. Cancer Res 2009; 69: 8742-8751.

44. Borin TF, Arbab AS, Gelaleti GB, et al. Melatonin decreases breast cancer metastasis by modulating ROCK-1 expression. J Pineal Res 2016; 60: 3-15.

45. Ahmed S, Harb OA, Nawar N. Prognostic Implications of Claudin 4 and Rock 1 in Triple Negative Breast Cancer. J Cancer Treat Res 2017; 5: 95-103.

46. Croft DR, Olson MF. The Rho GTPase effector ROCK regulates cyclin A, cyclin D1, and p27Kip1 levels by distinct mechanisms. Mol Cell Biol 2006; 26: 4612-4627.

47. Zhang C, Zhang S, Zhang Z, et al. ROCK has a crucial role in regulating prostate tumor growth through interaction with c-Myc. Oncogene 2014; 33: 5582-5591.

48. Huang Q, Qin S, Yuan X, et al. Arctigenin inhibits triple-negative breast cancers by targeting CIP2A to reactivate protein phosphatase 2A. Oncol Rep 2017; 38: 598-606.

49. Tseng L, Liu C, Chang K, et al. CIP2A is a target of bortezomib in human triple negative breast cancer cells. Breast Cancer Res 2012; 14: R68.

50. Wei L, Surma M, Shi S, et al. Novel Insights into the Roles of Rho Kinase in Cancer. Arch Immunol Ther Exp (Warsz) 2016; 64: 259-278.

51. Matsubara M, Bissell MJ. Inhibitors of Rho kinase (ROCK) signaling revert the malignant phenotype of breast cancer cells in 3D context. Oncotarget 2016; 7: 31602-31622.

52. Chang F, Zhang Y, Mi J, et al. ROCK inhibitor enhances the growth and migration of BRAF-mutant skin melanoma cells. Cancer Sci 2018; 109: 3428-3437.

\section{Address for correspondence}

\section{Nisreen Abdel Tawab Osman}

Pathology Department

Faculty of Medicine

Minia University

Minia, Egypt

e-mail: nisr20032000@yahoo.com 